\title{
Sequencing of superbugs seen as key to combating their spread
}

HINXTON, UK-Although the toll from hospital-acquired infections in the UK has declined in recent years from a high of close to 10,000 deaths in 2007 to around half that number today, the recurrent outbreaks are still troubling enough to merit alarm bells around the world. Part of the problem faced by hospitals is that current diagnostic methods have not kept pace with the need for rapid public health interventions, such as moving patients to isolation wards or emptying and deep-cleaning contaminated wards. To help bring clinical management up to speed, a new British consortium has embarked on an ambitious project to translate advances in gene sequencing technologies into diagnostic tools for tracking the spread of infections and informing doctors how to contain outbreaks in near to real time.

The Modernising Medical Microbiology Consortium, a five-year $£ 5.6$ million ( $\$ 8.7$ million) effort funded by the UK Clinical Research Collaboration, is focused on four major pathogens: Staphylococcus aureus (including methicillin-resistant (MRSA) strains), Clostridium difficile, norovirus and the tuberculosis bacterium. The researchers involved in the project-who hail from the UK Health Protection Agency, the University of Oxford and the Wellcome Trust Sanger Institute-envision a day when DNA from a sick person can be sequenced and compared with samples in a database to determine whether he or she brought the infection into the hospital or acquired it on the ward, possibly because of poor staff hygiene.

The initiative's first published success came earlier this year when a team led by Oxford microbiologist Kate Dingle created a Webaccessible database of $C$. difficile strains based on sequencing a series of housekeeping genes, and showed that DNA can be extracted directly from stools within days without an intermediate culturing step (J. Clin. Microbiol. 48, 770-778, 2010).

Building on this research, Oxford's Rosalind Harding reported here at the Infectious Disease Genomics and Global Health conference that sequencing whole $C$. difficile genomes could reveal the pattern and timing of a particular strain as it spread within a hospital ward. As validation of this approach, the genome-based reconstruction of transmission patterns also gelled with independent epidemiological data showing that the people with direct transmissions had crossed paths on the hospital ward. "This is like on the scale of criminal forensics," says Harding.
At the same meeting, Rory Bowden, a mathematical geneticist at Oxford, presented whole $S$. aureus genome data for more than 150 strains taken from two hospitals at two different time points each. Of note, the genomic data revealed a common ancestry among MRSA strains from a recent outbreak at the Brighton and Sussex University Hospitals NHS Trust, which had the worst MRSA infection rate in the country in 2006. This indicates that a particularly nasty form of MRSA must have swept through the wards, Bowden notes. So, despite the hospital's efforts to stay sterile by, among other measures, banning doctors from wearing neckties, the genomic data suggest that "it's perhaps less likely that the outbreak arose because of bad hygiene and more likely that it's just bad luck," he says.

Although these genomic approaches currently show promise for historical data, Ryan
Tewhey from the Scripps Research Institute in La Jolla, California says "there are still huge time lags before this will have clinical utility" in real time. Tewhey, who presented the genomes of 36 isolates of community-acquired MRSA at the meeting, notes that even though bacterial genomes can currently be sequenced for $\$ 100$, he estimates that we are still five to ten years away from getting these genomes sequenced and assembled in a matter of days instead of weeks.

Even though the hardware is not yet in place to sequence deadly microbes on the spot in hospital settings, scientists should work now to refine the computational tools that instantly analyze such DNA tests, says Tanya Golubchik, an Oxford bioinformaticist who is part of the initiative. "We want to be there when it happens," she says.

Elie Dolgin

\section{Genomics uncovers microbe resistance}

HINXTON, UK-In May 2004, a 64-year-old woman with a hemodialysis catheter entered a community hospital in Indiana with traces of the bacterium Enterococcus faecalis in her blood. Testing revealed the strain to be ampicillin susceptible but vancomycin resistant. Because of the woman's history of penicillin allergy, she was treated with another antibiotic, linezolid, to clear the infection. Not long afterward, her platelet count dropped dangerously low, so doctors switched her to yet another antibiotic-oral ciprofloxacin-and discharged her. A few days later, she returned to the hospital with a fever and was put on daptomycin, a last line of defense for the management of drug resistant Gram-positive bacterial infections, and again sent home. Just two weeks later, she came back with signs of daptomycin-resistant bacteria, and she died soon thereafter (Clin. Infect. Dis. 41, 565-566, 2005).

What happened? To help put together the pieces, a team led by Cesar Arias, an infectious diseases physician at the University of Texas Medical School in Houston, turned to faster 'next generation' genetic sequencing. Reporting at the Infectious Diseases Genomics and Global Health meeting here last month, Arias's team found that the microbe that had ultimately killed the woman had acquired a handful of mutations that rendered it immune to daptomycin-the first time that an $E$. faecalis infection gained spontaneous daptomycin resistance while a patient was receiving therapy. (In 2004, less than a year after daptomycin first became available in the US, clinicians also observed the emergence of daptomycin resistance in patients with Enterococcus faecium and those with methicillinresistant Staphylococcus aureus.)

Several of the mutations seemed to affect the bacterial cell's physiology, including changes that prevented daptomycin from inserting into the cell membrane, reported Arias. He is now using a rat model of infection to see if he can replicate the in vivo evolution of antibiotic resistance with an eye to developing new compounds to prevent resistance from developing. "If we understand how this works, then it'll open up a Pandora's box of drug discovery," Arias says.

The need for new drugs could not be more urgent, especially for E. faecalis, a highly virulent microbe known for its capacity to transfer antibiotic resistance to other pathogens. "If we lose the 